\title{
Dynamics of Traveling Waves in the Transverse Section of a Laser
}

\author{
Eamonn O’Neill, * John Houlihan, John G. McInerney, and Guillaume Huyet \\ Physics Department and Tyndall National Institute, Lee Maltings, National University of Ireland, University College Cork, Ireland
}

(Received 17 May 2004; published 14 April 2005)

\begin{abstract}
We analyze the general features of the formation and interaction of transverse traveling waves and the appearance of filamentation in broad area semiconductor lasers with current profiling. For small apertures, the emitted profile is symmetric consisting of two counterpropagating transverse traveling waves, both emanating from the center of the device. For larger apertures, the emission becomes asymmetric as one of the traveling waves expands to occupy an increased area while the other occupies the remaining, smaller spatial region. In both devices, the pattern becomes unstable at higher injection currents due to optical filamentation, although an intermediate state is present in the wider device whereby the dominant wave undergoes a Hopf bifurcation before filamentation occurs.
\end{abstract}

DOI: 10.1103/PhysRevLett.94.143901

PACS numbers: 42.65.-k, 42.55.Px

Pattern formation and the evolution of complexity in spatially extended dynamical systems has been the subject of many investigations in widely diverse branches of science. In nonlinear optics [1], such studies have been motivated by the goal of developing a general understanding to describe the emergence of periodic [2,3] and quasiperiodic patterns [4], vortices [5,6] or spatial solitons [7] using various passive nonlinear optical systems as well as gas [8$11]$ and semiconductor lasers [3,12-14]. The analysis of the transverse structure of the light emitted by large aperture semiconductor lasers is extremely interesting due to the strong phase-amplitude coupling of the active material, as parametrized by the $\alpha$ factor [15]. In these laser devices, a propagating beam decreases the local carrier density, which induces an increase of the refractive index and thus results in a nonlinear waveguide which focuses the light and induces further carrier depletion and index change. This phenomenon, which is at the origin of the appearance of instabilities, leads to the deterioration of the spatial coherence of broad area semiconductor lasers and limits their suitability for many potential applications. Although such instabilities have been widely analyzed, both experimentally and theoretically, there are few fundamental insights into the evolution of these instabilities in semiconductor lasers.

The aim of this paper is to analyze the onset of transverse instabilities in broad area semiconductor lasers as the transverse aperture is increased. The devices used in this paper include a special current spreading layer to enhance carrier diffusion and generate a smooth injection current profile [16]. Thus, by designing devices with different contact stripe widths, we can analyze the appearance of filamentation as the transverse section of the laser is increased. For small stripe widths, $<10 \mu \mathrm{m}$, the pump profile can be described by a bell function, such as a Lorentzian, and the optical field is described by two counterpropagating transverse waves emitted from the center of the devices, resulting in symmetric off-axis emission. Wider stripes, $>20 \mu \mathrm{m}$, result in a smoothed current pro- file at the edge while remaining constant in the center region (see Fig. 1). In this case, the emission becomes asymmetric as one wave becomes dominant. Increasing the pump parameter leads to the emergence of an oscillatory state before the observation of spatiotemporal chaos at high pump levels.

From a theoretical viewpoint, if the carrier dynamics can be adiabatically eliminated, the spatiotemporal dynamics of wide aperture semiconductor lasers can be described by the complex Ginzburg-Landau equation $[5,17,18]$,

$$
\partial_{t} E=\kappa(1-i \alpha)\left[J(x)-1-|E|^{2}\right] E+i \kappa \frac{a}{2} \nabla^{2} E,
$$

where $E$ is the complex amplitude of the slowly varying electric field, $\kappa$ is the rate of electric field damping (due to the absorption of the semiconductor medium and mirror losses), $J(x)$ is the normalized pump parameter, $\nabla^{2}$ is the transverse Laplacian, $a$ is the diffraction parameter, and $\alpha$ parametrizes the carrier dependent refractive index. The complex Ginzburg-Landau equation usually contains a diffusive term, but this term can be neglected in semiconductor lasers as it is proportional to the curvature of the gain spectrum [5]. However, for semiconductor lasers, the carrier lifetime, $1 / \gamma_{n}$, is much larger than the photon life-

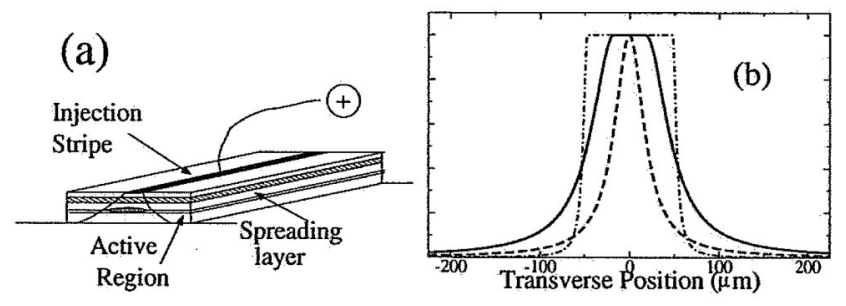

FIG. 1. (a) Schematic of the broad area laser with spreading layer. (b) Current profiles used for modeling narrow, $5 \mu \mathrm{m}$ wide, stripe device, termed narrow stripe injection (dashed), wide, $50 \mu \mathrm{m}$, stripe device, termed wide stripe injection (solid) and, for comparison, a usual broad area laser (dot-dashed). 
time and a more complete description which takes into account the carrier density dynamics $N(t)$ reads

$$
\begin{aligned}
\partial_{t} E & =\kappa(1-i \alpha)(N-1) E+i \kappa \frac{a}{2} \nabla^{2} E, \\
\partial_{t} N & =-\gamma_{n}\left[N-J(x)+N|E|^{2}\right] .
\end{aligned}
$$

These equations have been extended to provide a more accurate description of the behavior of broad area semiconductor lasers by incorporating the full gain curve as calculated from microscopic many-body theory [19]. Indeed, since the essential features of the experimental results presented in this paper can be captured by Eqs. (1) and (2) and in the Maxwell-Bloch equations [16] they should also be observed in these more sophisticated models. The steady-state solutions of (1) and (2) are unstable when $J(x)$ is flat due to the Benjamin-Feir instability [17]. Experimentally, this is demonstrated in regular broad area lasers, which have top hat shaped current profiles
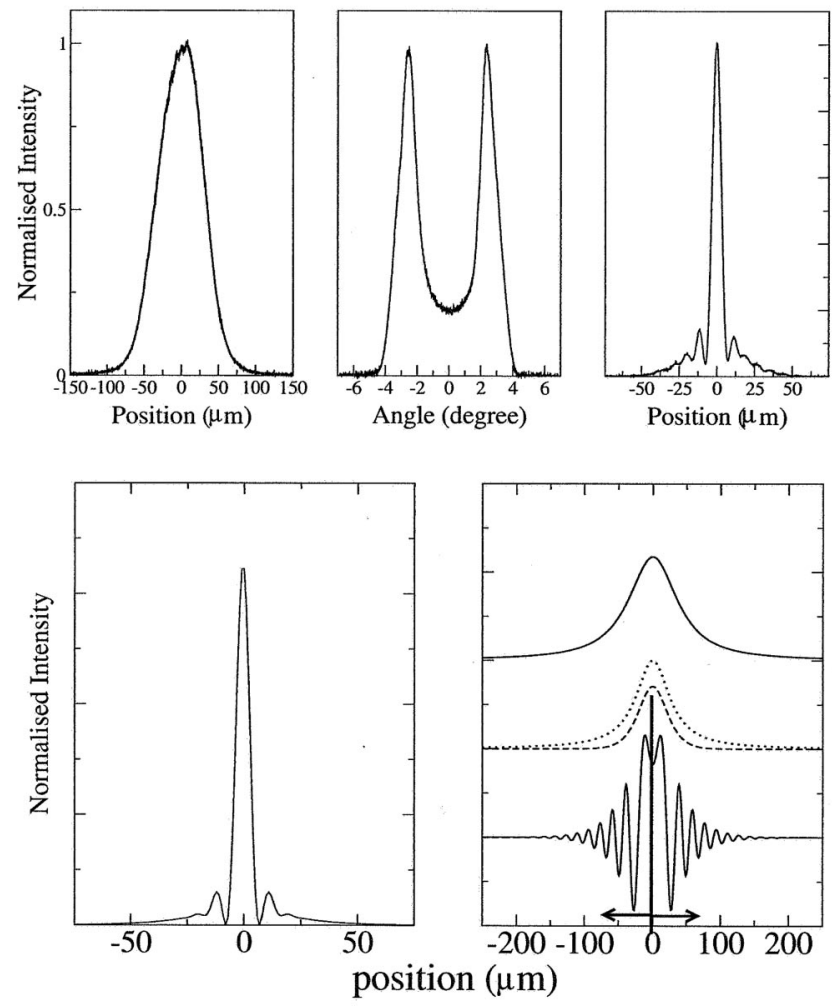

FIG. 2. Experimental near (top left) and far (top center) fields and beam waist (top right) of a $5 \mu \mathrm{m}$ stripe device under pulsed operation. Transverse traveling waves counterpropagate from the center of the near field profile to the edges and result in the dual lobed far-field pattern. Also, Ginzburg-Landau simulations showing the beam waist (bottom left) and (bottom right, from top to bottom) the corresponding pump profile, carrier profile, intensity profile, and real part of the electric field for a narrow stripe injection profile (see Fig. 1 for further details). The parameters used were $\kappa=1$ (time unit in the model $=1 \mathrm{ps}$ ), maximum of $J(x)$ was $1.34, a=1$ (the length unit in the model is $5 \mu \mathrm{m})$, and $\alpha=1.2$. resulting in the appearance of these instabilities and consequently poor spatial coherence properties. In addition, the stability of transverse traveling wave solutions in equations similar to (2) have been addressed in [20] and some control schemes developed.

The broad area semiconductor lasers studied in this work were fabricated from a standard graded index separate confined heterostructure with an additional $p$-type spreading layer (for further details see [16]). The inclusion of this layer, between the injection stripe and the active region of the laser, is a simple and effective method to achieve smooth edges in the transverse direction. By varying the thickness and doping concentration of the spreading layer, different contact stripe widths give different profiles; here we present stripe widths of 5 and $50 \mu \mathrm{m}$ which correspond to the two profiles illustrated in Fig. 1. In order to observe the carrier-field interaction, without the interference of thermal guiding effects, the devices were electrically driven under pulsed conditions at $1 \mathrm{kHz}$ with a duty cycle less than $0.01 \%$. The near field of the laser was obtained by imaging the laser facet, while the far field was measured after free space propagation. The beam waist of the laser was located and measured by scanning the transverse profile of the laser at different points along the propagation direction. This last measurement provides a quick and reliable measurement of the spatial coherence of the device when the device far-field divergences are similar, as spatially coherent devices have narrow beam waists while a broad time-averaged beam waist may be associated with spatiotemporal instabilities in the laser beam. In the lateral (growth) direction the epitaxial structure ensured single mode operation.

For the narrow stripe lasers, i.e., when the current profile can be approximated by a smooth bell function, the near field follows the pump profile while the far field contains symmetrical off-axis emission as shown in Fig. 2. The possibility of generating off-axis emission with such injection profiling was first identified by Petermann [21]. Experimentally, this off-axis emission was clearly demonstrated in [16] where the near field followed the injection profile while the far field contained two well defined lobes, symmetric about the optical axis. In addition, by filtering the far field of the laser and reimaging the near field, it was shown that each lobe of the far field is associated with onehalf of the near field. Thus, the optical field in the laser can be described in terms of two counterpropagating transverse traveling waves, where each wave originates in the center of the device and propagates towards the edge. Thus both waves sources are at the center while the sinks are on the edges of the injection profile. The small beam waist (less than $5 \mu \mathrm{m}$ ) and the presence of side lobes demonstrates a high degree of spatial coherence, and the emitted pattern can therefore be considered as a single but complicated transverse mode. This was previously explained using the framework of the Maxwell-Bloch equations but can also be 
described within the complex Ginzburg-Landau framework (see Fig. 2) as the smooth current profile creates a smooth antiguide due to the carrier-induced index reduction which stabilizes the emission. In both the experiment and simulation, the increase of the pump parameter beyond some critical value results in the onset of instabilities. In this situation, the time-averaged far field remains double lobed in average; however, the beam waist broadens dramatically as the onset of spatiotemporal chaos results in a reduction in the spatial coherence of the beam.

For devices with stripes wider than $50 \mu \mathrm{m}$, the far field remains double lobed at low injection currents, however, the intensity of one peak is stronger than the other (see Fig. 3). This indicates that the symmetry observed for narrow devices has been broken, and that one traveling wave has become dominant in the laser emission. Such loss
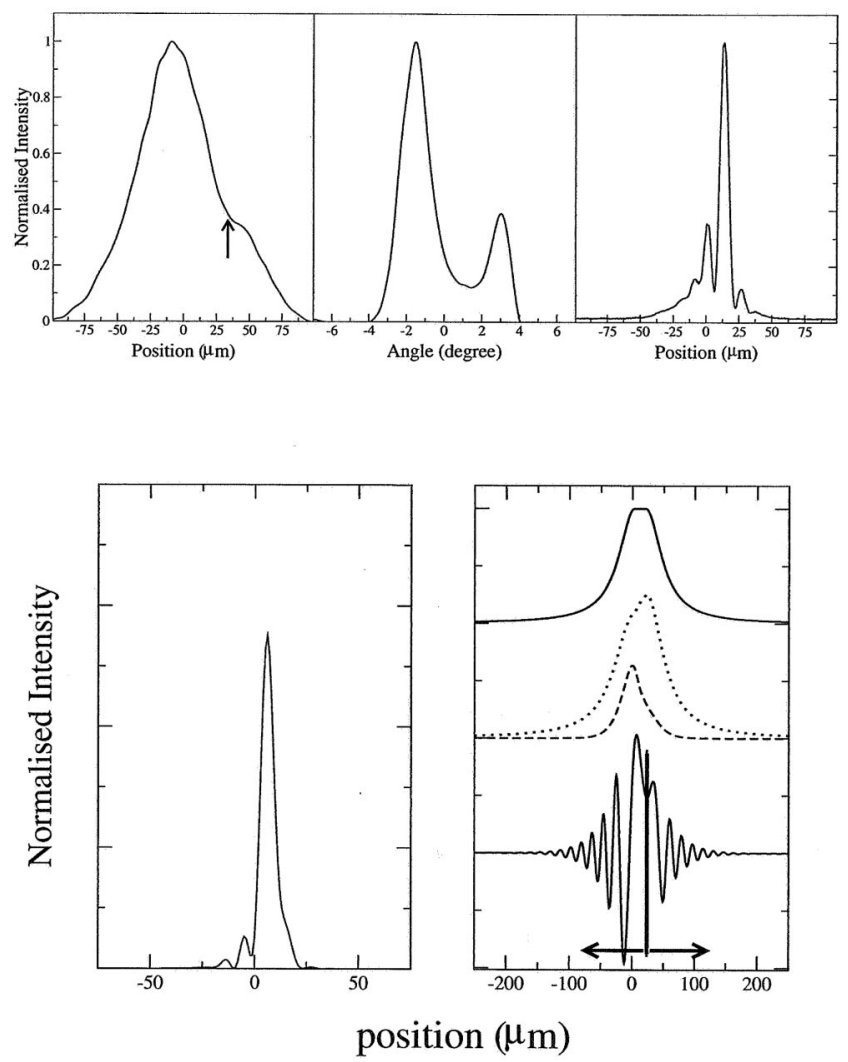

FIG. 3. Experimental near (top left) and far (top center) fields and beam waist (top right) of a $50 \mu \mathrm{m}$ stripe device under pulsed operation displaying the presence of stable transverse traveling waves. Note that, in this case, one of the traveling waves has become dominant as apparent in the asymmetry in the far-field profile. Also, Ginzburg-Landau simulations showing the beam waist (bottom left) and (bottom right, from top to bottom) the corresponding pump profile, carrier profile, intensity profile, and real part of the electric field for a wide stripe profile (see Fig. 1 for further details). The parameters used were the same as used in Fig. 2. Note that the position of the source has moved to the edge of the profile and that a shoulder has appeared in the near field as in the experiment. of symmetry is also apparent in the near field, where an unusual shoulder appears on one side of the profile. The reimaged beam waist remains narrow, indicating a high degree of spatial coherence, and continues this trend of symmetry breaking, having its maximum off axis. Numerical simulations of both the Ginzburg-Landau and the Maxwell-Bloch equations also reveal the existence of symmetry breaking when the transverse dimension of the device is increased (see Fig. 3). In this situation, the numerical simulations exhibit a monochromatic solution where the source of the waves has moved from the center
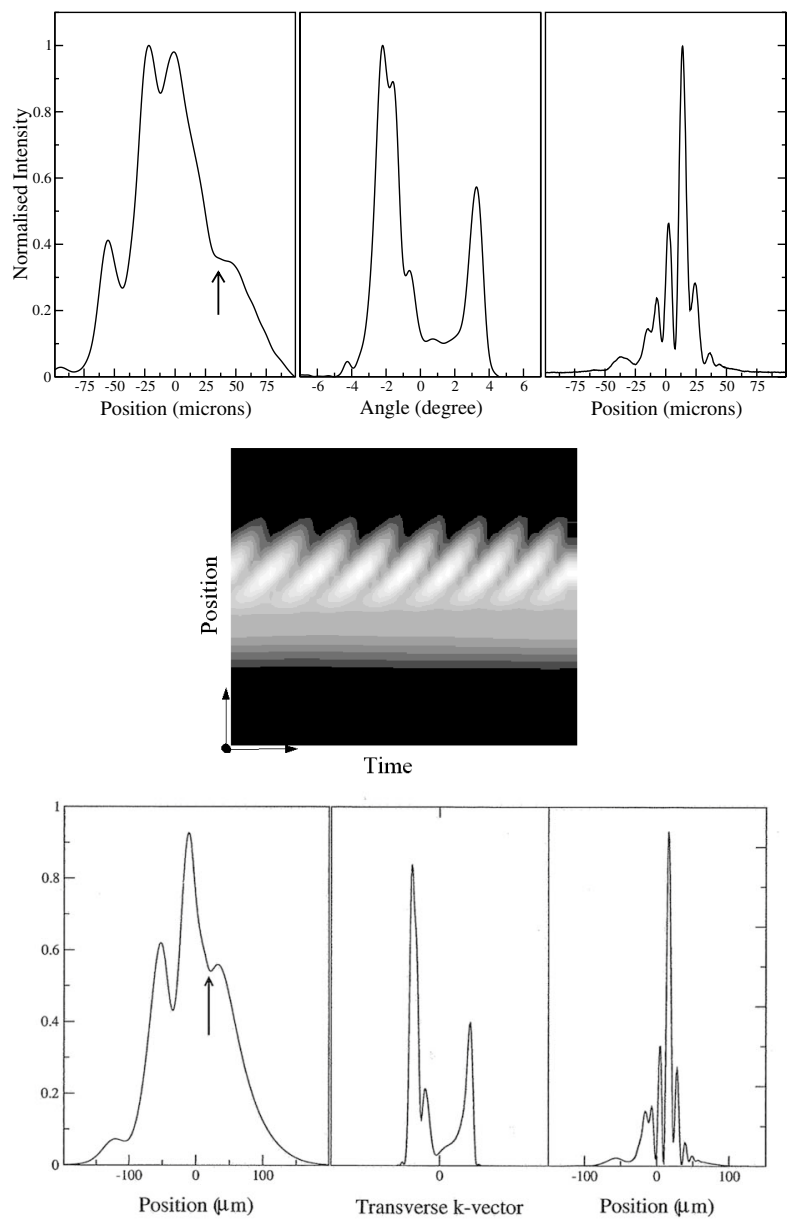

FIG. 4. Experimental near (top left) and far (top center) fields and beam waist (top right) of a $50 \mu \mathrm{m}$ stripe device at increased injection displaying modulation in the dominant wave side of the near and far fields, indicating some instability. Note, however, that the beam waist remained narrow indicating that the laser emission was regular. Simulation results of the rate equation model showing a density plot of the near field intensity as a function of time (center) and instantaneous near (bottom left) and far fields (bottom center) and beam waist (bottom right) for a wide stripe profile. The parameters used were $\kappa=1$ (time unit in the model $=1 \mathrm{ps),} \alpha=4, \quad a=1$ (length unit in the model $=5 \mu \mathrm{m}), \gamma_{\|}=5 \times 10^{-3}$. Note the self-pulsing dynamics in the near field evolution and the good comparison with experiment. 
of the injection profile to the boundary between the flat central region and the smoothened edges as shown in Fig. 3. This position is identifiable in the near field intensity profile by the appearance of a shoulder, similar to that observed in the experiments. Numerically, the direction of the dominant traveling wave (or equivalently the position of both waves source) varies from run to run depending on the initial conditions. This is not observed in the experiment, probably due to the presence of local inhomogeneities and impurities in the device which break the symmetry of the system and slightly favor one direction.

Experimentally, when the pump parameter was increased, a spatial modulation appeared on the dominant wave side of both the near and far-field profiles, as shown in Fig. 4, suggesting that some destabilization of the dominant traveling wave has occurred. The fact that the beam waist remains narrow indicates that the laser emission remains regular and not chaotic. Maxwell-Bloch simulations at increased injection levels display similar features in the instantaneous near and far fields (Fig. 4), which correspond to a destabilization of the dominant traveling wave to an oscillatory state. However, such modulation averages out and does not appear in the time-averaged field as in the experiment. Again, the role of imperfections and inhomogeneities is suggested here as a possible mechanism for the residual modulation in the experimental near field. Numerically, in this spatial region the emission undergoes self-pulsing dynamics, while in the region containing the recessive traveling wave it remains stable. Remarkably, the beam waist remains narrow and stable in time indicating no loss in spatial coherence of the beam. If the pump is further increased, filamentary dynamics ensue in both experimental and numerical situations and result in a dramatic broadening of the laser's beam waist.

In summary, these results show that spatially varying parameters can inhibit the appearance of self-focusing instabilities. Variations in the pump profile, for example, stabilize traveling wave solutions whose sources/sinks are localized at the maxima/minima of the spatial variations. For small stripe widths, this corresponds to the symmetric pattern observed in the narrow stripe lasers. If the gradient becomes weak in the center of the device, as in the wider injection profile, then one traveling wave dominates and the spatial symmetry is broken. The source position changes to follow the maximum of the spatial variation which now occurs at the interface between the flat central region and decaying edge regions. At higher injection levels, the dominant wave becomes unstable through a Hopf bifurcation before developing spatiotemporal chaos.
We gratefully acknowledge James O'Callaghan for the fabrication of the devices. This was supported by Science Foundation Ireland under contract no. /01/fi/co13.

*Present address: Fab24, Intel, Leixlip, Co. Kildare, Ireland.

[1] F. T. Arecchi, S. Boccaletti, and P. L. Ramazza, Phys. Rep. 318, 1 (1999).

[2] A. V. Mamaev and M. Saffman, Opt. Commun. 128, 281 (1996).

[3] S.P. Hegarty, G. Huyet, J. G. McInerney, and K. D. Choquette, Phys. Rev. Lett. 82, 1434 (1999).

[4] E. Pampaloni, P. L. Ramazza, S. Residori, and F. T. Arecchi, Phys. Rev. Lett. 74, 258 (1995).

[5] P. Coullet, L. Gil, and F. Rocca, Opt. Commun. 73, 403 (1989).

[6] D. Rozas, Z. S. Sacks, and G. A. Swartzlander, Jr., Phys. Rev. Lett. 79, 3399 (1997).

[7] S. Barland, J. R. Tredicce, M. Brambilla, L. A. Lugiato, S. Balle, M. Giudici, T. Maggipinto, L. Spinelli, G. Tissoni, T. Knodl, M. Miller, and R. Jager, Nature (London) 419, 699 (2002).

[8] C. K. Green, G. B. Mindlin, E. J. D’Angelo, H. G. Solari, and J. R. Tredicce, Phys. Rev. Lett. 65, 3124 (1990).

[9] D. Dangoisse, D. Hannequin, C. Lepers, E. Louvergneaux, and P. Glorieux, Phys. Rev. A 46, 5955 (1992).

[10] G. Huyet, C. Martinoni, J. R. Tredicce, and S. Rica, Phys. Rev. Lett. 75, 4027 (1995).

[11] F. Encinas-Sanz, I. Leyva, and J. M. Guerra, Phys. Rev. Lett. 84, 883 (2000).

[12] I. Fischer, O. Hess, W. Elsasser, and E. Gobel, Europhys. Lett. 35, 579 (1996).

[13] K. F. Huang, Y. F. Chen, H. C. Lai, and Y. P. Lan, Phys. Rev. Lett. 89, 224102 (2002).

[14] Y. F. Chen, K. F. Huang, H. C. Lai, and Y. P. Lan, Phys. Rev. Lett. 90, 053904 (2003).

[15] M. Osinski and J. Buus, IEEE J. Quantum Electron. 23, 9 (1987).

[16] V. Voignier, J. Houlihan, J. R. O'Callaghan, C. Sailliot, and G. Huyet, Phys. Rev. A 65, 053807 (2002).

[17] I. S. Aranson and L. Kramer, Rev. Mod. Phys. 74, 99 (2002).

[18] R. Lefever, L. A. Lugiato, W. Kaige, N. B. Abraham, and P. Mandel, Phys. Lett. A 135, 254 (1989).

[19] M. Kolesik and J. V. Moloney, IEEE J. Quantum Electron. 37, 936 (2001).

[20] G. K. Harkness, G.-L. Oppo, R. Martin, A. J. Scroggie, and W. J. Firth, Phys. Rev. A 58, 2577 (1998).

[21] K. Petermann, Opt. Quantum Electron. 13, 323 (1981). 